\title{
DISPOSITIVO PARA AUXILIAR ENSINO EM ENGENHARIA AEROESPACIAL
}

Pedro Jorge S. Correa, Victor Henrique C. Barbosa e Manuel N. D. Barcelos Júnior

RESUMO: Devido à grande quantidade de disciplinas teóricas disponíveis no Curso de Engenharia Aeroespacial da Faculdade do Gama, UnB, tem-se um distanciamento entre o que é ministrado na sala de aula com sua aplicação prática. A construção de um dispositivo de teste modular, confiável e de baixo custo dedicada ao exercício dos conhecimentos teóricos apreendidos nas disciplinas desse curso visa apresentar uma possível solução para minimizar essa dificuldade.

Palavras-chave: Dispositivo de Testes, Laboratório Móvel, Ensino Aplicado, Educação em Engenharia Aeroespacial.

ABSTRACT: Due to the great number of theoretical classes in the Course of Aerospace Engineering of the University of Brasília at Gama, a distance exists between what is taught at the classroom and the practical application. The construction of a modular, reliable and low cost test bench dedicated to the exercise of theoretical knowledge apprehended in the classes of the course intends to present a possible solution to minimize this difficulty.

Keywords: Test Device, Mobile Laboratory, Applied Teaching, Aerospace Engineering Education.

RESUMEN: Debido a la gran cantidad de materias teóricas disponibles en el Curso de Ingeniería Aeroespacial de la Universidad de Brasília en Gama, hay una brecha entre lo que se enseña en el aula y la aplicación práctica. La construcción de un dispositivo de prueba modular, fiable y de bajo coste dedicado al ejercicio de los conocimientos teóricos adquiridos en las asignaturas de este curso es dar a conocer una posible solución para minimizar esta dificultad.

Palabras clave: Dispositivo de prueba, Laboratorio móvil, Enseñanza aplicada, Educación en ingeniería aeroespacial.

\section{INTRODUÇÃO}


Uma das grandes dificuldades enfrentadas por cursos de engenharia em universidades brasileiras é a interação entre o conhecimento prático e teórico. Esse problema é mais evidente em cursos de engenharia porque formam profissionais dos quais o mercado de trabalho exige o chamado know-how, que consiste no conhecimento técnico aliado à prática. Entretanto, a prática de engenharia tem um alto custo financeiro, de modo que mesmo as mais renomadas universidades brasileiras sofrem com a falta de equipamentos e laboratórios para o desenvolvimento de atividades práticas. $\mathrm{O}$ alto custo aplica-se não apenas aos equipamentos em si, mas também à manutenção desses equipamentos em operação, com os insumos necessários ao seu funcionamento.

Segundo uma reportagem da Confederação Nacional da Indústria (CNI) apenas $42 \%$ dos Engenheiros brasileiros atuam em sua área de formação (dados referentes a 2014). Um dos motivos para esse fato, conforme a reportagem, é a dificuldade do contato com situações práticas de aplicação do conhecimento em engenharia. Os engenheiros recém formados deixam as universidades com um vasto conhecimento teórico mas sem saber como aplicá-lo em situações reais. Como consequência, é comum que os engenheiros busquem outras áreas profissionais, por falta de domínio prático da sua área de formação. Outro assunto abordado foi o ensino de Engenharia em países com grande desenvolvimento como a Alemanha, onde, de acordo com a reportagem, o ensino é voltado para a prática e a aplicação na indústria [1].

Tendo em vista as dificuldades citadas acima, o dispositivo desenvolvido tem por objetivo principal amenizar a dificuldade prática dos graduandos em engenharia aeroespacial. O equipamento e materiais utilizados são de baixo custo, fácil obtenção e manutenção, de modo a viabilizar seu uso no ambiente acadêmico. Obviamente apenas um dispositivo não é suficiente para findar tais dificuldades, mas ele representa uma maneira de inovar na busca por soluções para o ensino prático de engenharia no Brasil.

\section{O DISPOSITIVO}

O dispositivo consiste em um VANT (Veículo Aéreo Não Tripulado) quadrotor construído utilizando equipamentos de prateleira (equipamentos encontrados facilmente no mercado) e de baixo custo. Seus componentes são: um frame de um quadrotor, uma placa de

arduino uno, bateria, quatro motores, quatro hélices, quatro controladores eletrônicos de velocidade (ESC, do Inglês Electronic Speed Control), módulo giroscópio e módulo bluetooth (conexão de rede sem fio).

\subsection{MATERIAIS}


A estrutura do dispositivo consiste em um frame F450, desenvolvido pela DJI. Com esta plataforma, é possível adaptar diversos tipos de componentes e sensores, pois ela é simples e de fácil montagem. A Figura 1 mostra o frame.

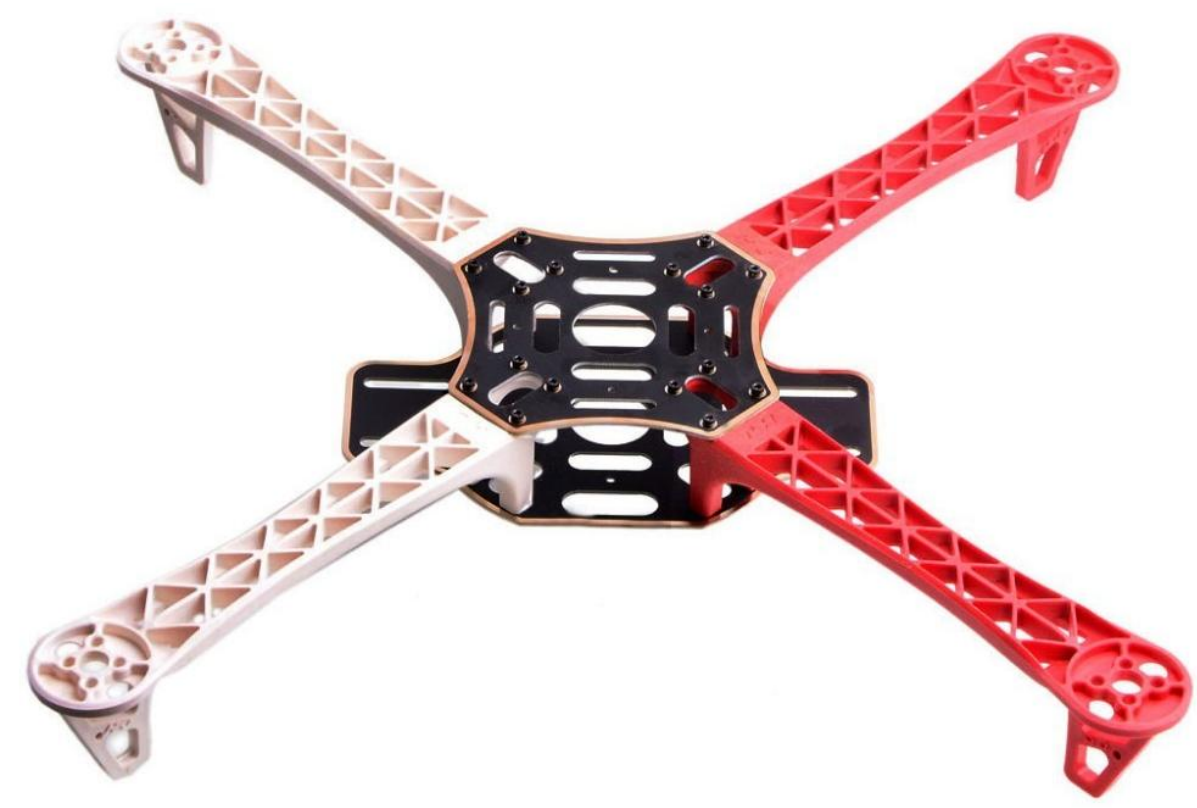

Figura 1: Frame F450, utilizado como componente estrutural no dispositivo (Fonte: [2])

A figura 2 mostra as dimensões do frame, para uma melhor compreensão da estrutura do dispositivo.
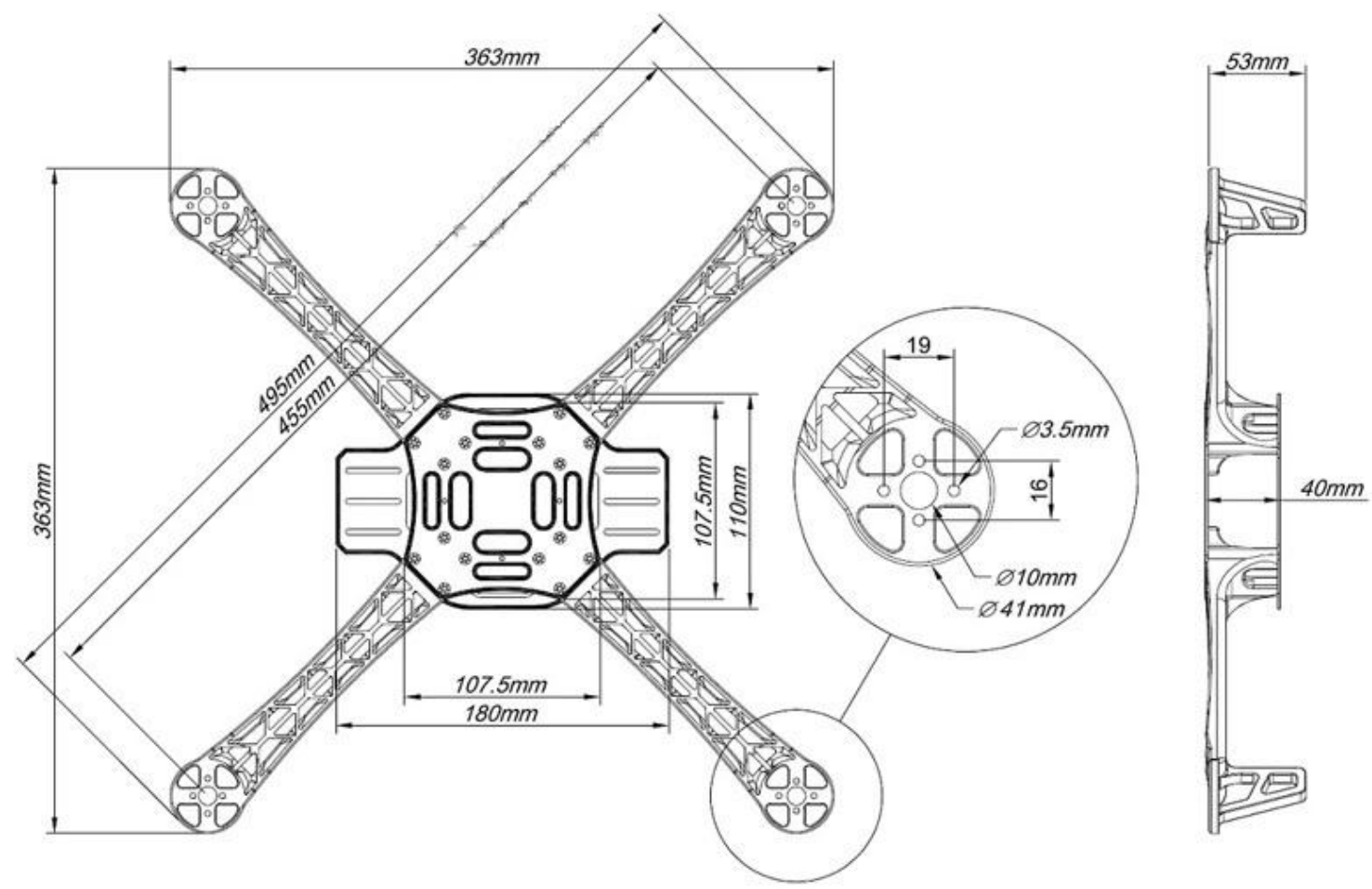

Figura 2: Dimensões do frame F450 (Fonte: [3]) 
A placa controladora escolhida foi uma Arduino UNO, devido a seu baixo custo e por ser totalmente programável. Este tipo de placa é facilmente encontrada no mercado. A figura 3 mostra uma placa Arduino UNO.

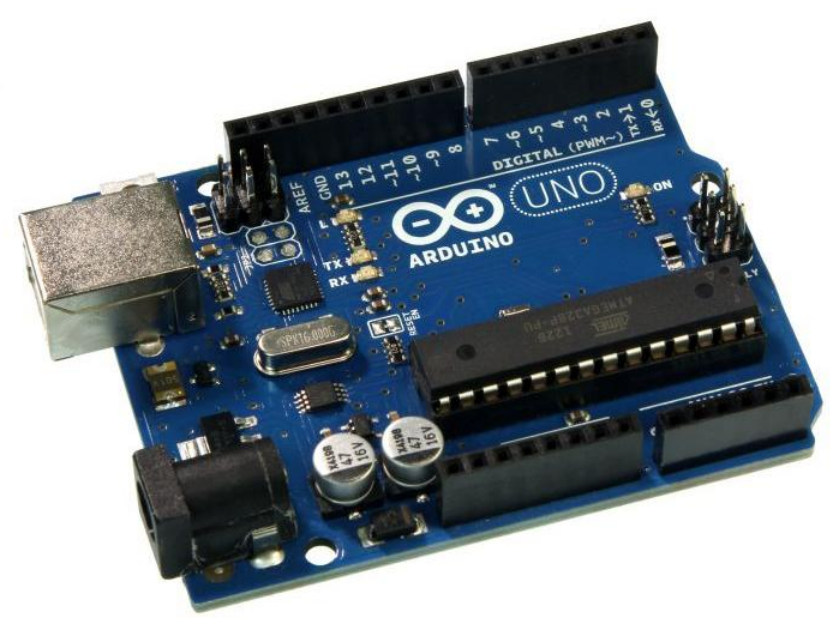

Figura 3: Placa Arduino UNO (Fonte: [4])

O conjunto moto-propulsor, formado por hélices e motores, é escolhido de acordo com as características desejadas para o dispositivo, como capacidade de carga útil e velocidade de voo. Os motores utilizados são do tipo brushless (sem escovas) e as hélices são escolhidas em função das características do motor. Os ECS, por sua vez são os responsáveis pela mudança na velocidade dos motores, fazendo a comunicação entre a placa controladora e os motores. A figura 4 mostra um conjunto moto-propulsor com um esc.

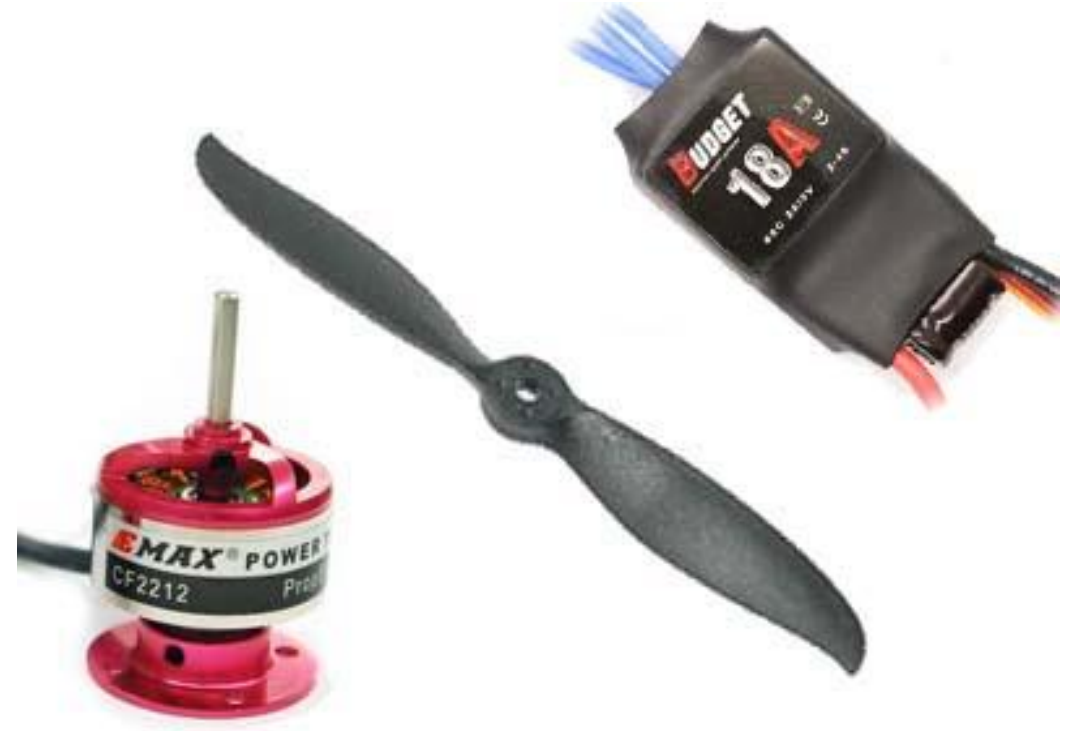

Figura 4: Motor brushless, hélice e ESC (Fonte: [5]) 
Para a determinação da atitude do VANT, faz-se necessário o uso de um sensor de medidas inerciais (IMU, do inglês Inertial Measurement Unit) compatível com o arduino. O sensor utilizado foi o MPU6050, que conta com um giroscópio e um acelerômetro, em 3 eixos. A figura 5 mostra o sensor IMU utilizado.

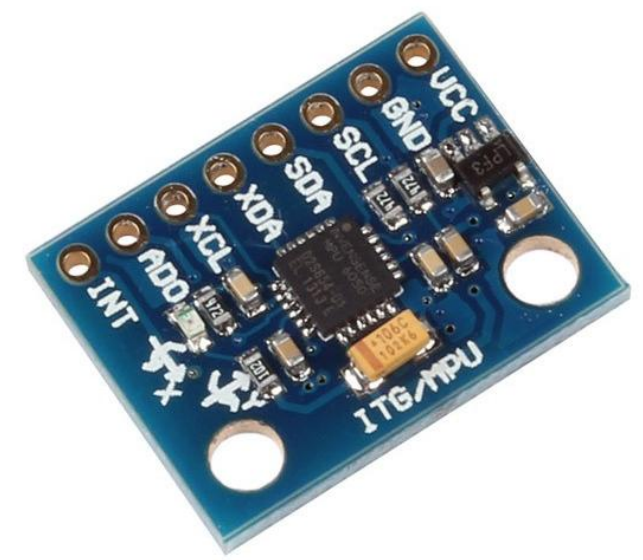

Figura 5: MPU6050, IMU com giroscópio e acelerômetro (Fonte: [6])

Por fim, a forma de comunicação escolhida foi o bluetooth, por ser barata e acessível, pois qualquer dispositivo com sistema operacional Android pode se comunicar com o módulo bluetooth. A comunicação bluetooth é de curto alcance, mas isso não se torna um problema, pois o dispositivo foi planejado para ser utilizado para testes e estudos, e não para voos a longas distâncias. A figura 6 mostra o módulo bluetooth utilizado (HC-05), também compatível com o arduino.

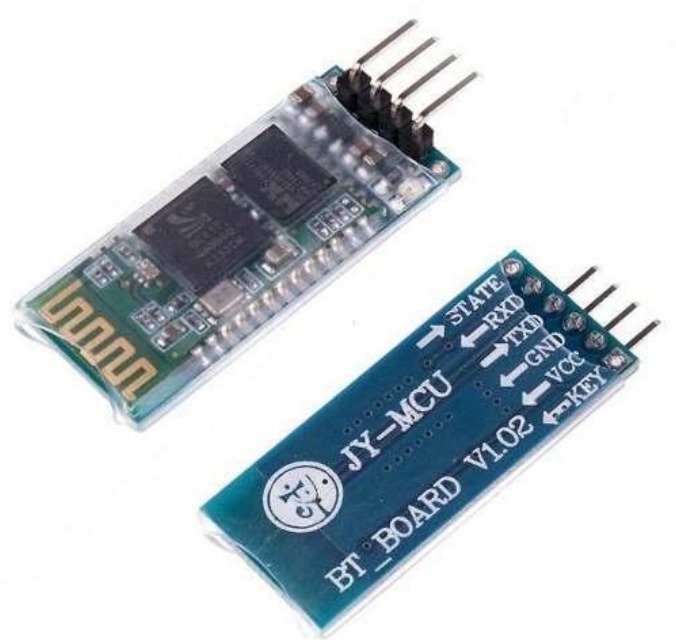

Figura 6: Módulo bluetooth utilizado (Fonte: [7])

O dispositivo montado, com os materiais citados, é mostrado na figura 7. 


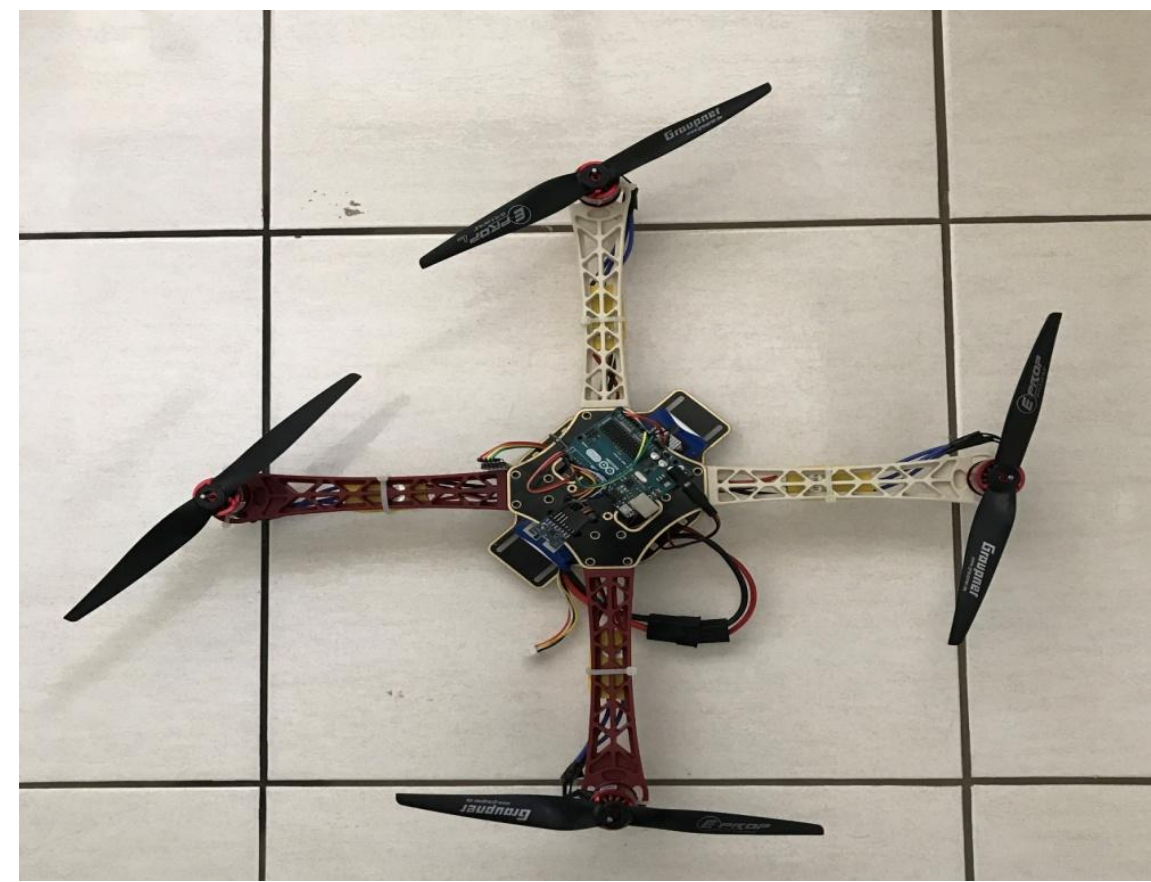

Figura 7: Dispositivo montado (Fonte: [elaborada pelos autores])

\subsection{SISTEMA DE CONTROLE}

Como uma forma de incentivo ao uso de plataformas mais interativas e úteis no ensino, o sistema de controle de atitude do drone foi completamente desenvolvido, simulado e implementado no Arduino Hardware utilizando-se o software MATLAB Simulink, da Mathworks [8]. Este software possibilita o desenvolvimento de todo o sistema de controle por meio de diagramas de blocos e gera automaticamente o código em linguagem $\mathrm{C}$, que é utilizado pelo arduino [9].

Para o desenvolvimento do sistema de controle, utilizou-se a teoria de controle PID (Proportional-Integral-Derivative), aproximando os parâmetros por tentativa e erro até que fosse atingida uma resposta satisfatória [10]. Os ângulos de referência utilizados para o controle foram os ângulos de Euler (rolagem, arfagem e guinada). A figura a seguir ilustra esses ângulos e o posicionamento do eixo de referência em relação ao quadrotor. 


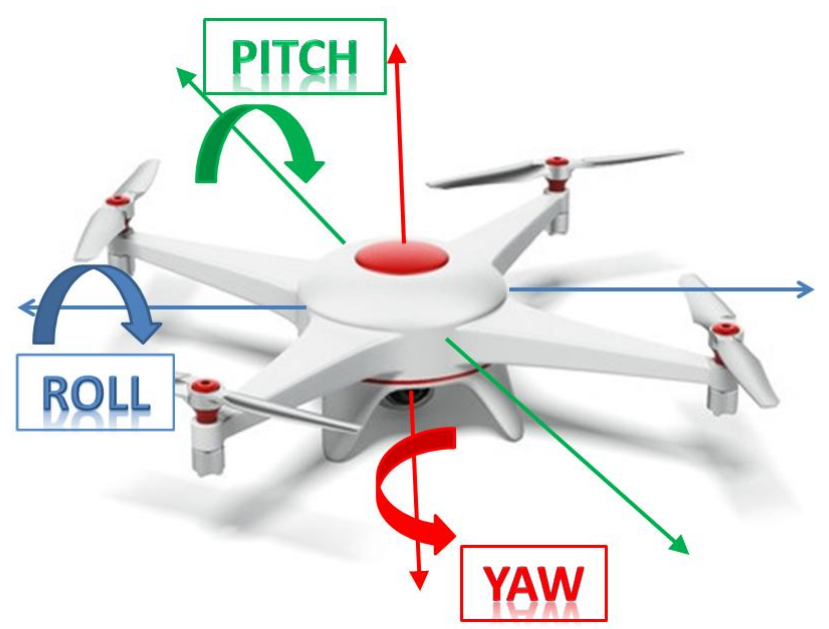

Figura 8: Ângulos de Euler em um quadrotor (Fonte: [11])

Para a realização das primeiras simulações, foram definidos ângulos iniciais para o roll e para o pitch e um ângulo desejado para ambos. As figuras a seguir mostram duas destas simulações, nas quais foram definidos ângulos iniciais de 20 e -15 graus para roll e pitch, respectivamente. Na figura 9, o ângulo desejado foi definido como 0 graus, enquanto que na figura 10 o ângulo desejado foi definido como 15 graus.

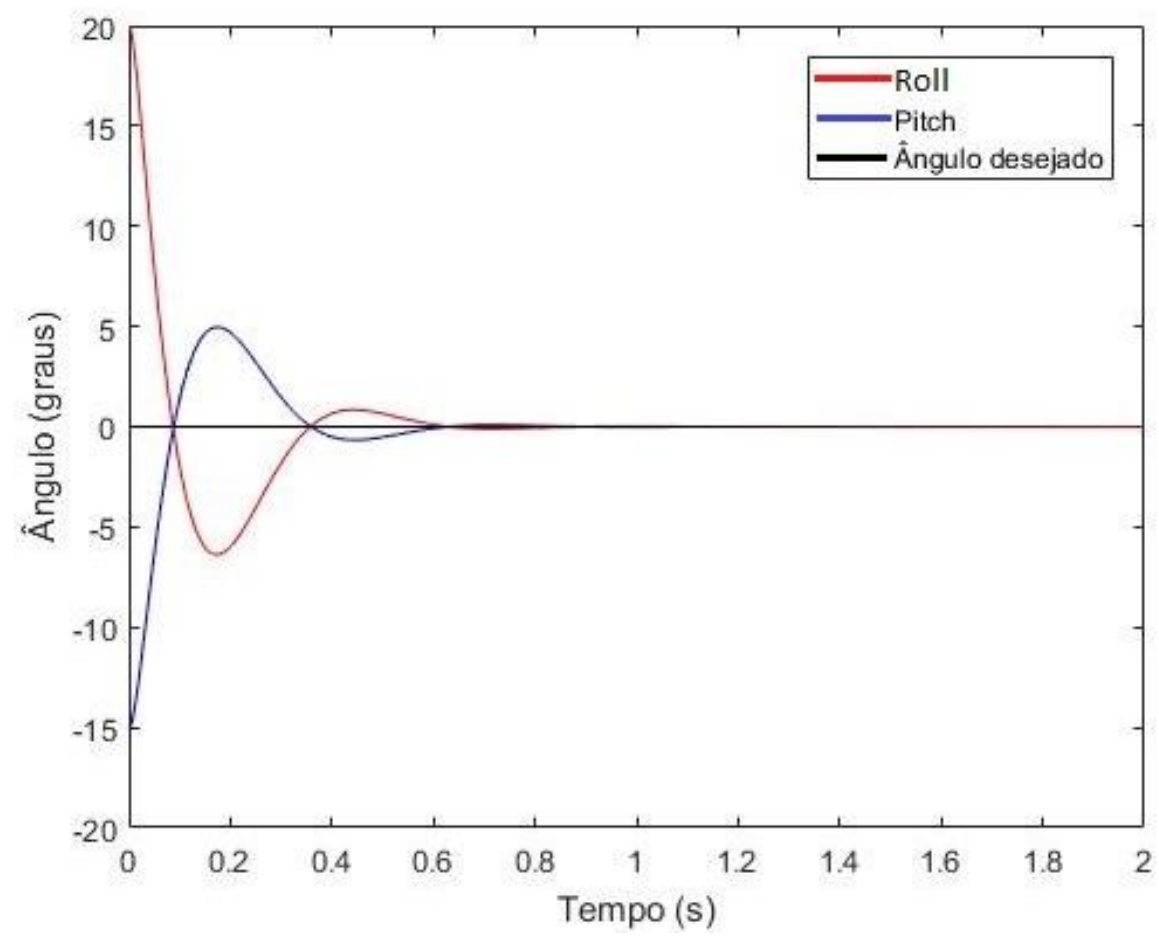

Figura 9 - Roll e pitch em função do tempo, com posição desejada de 0 graus (Fonte: elaborada pelos autores). 


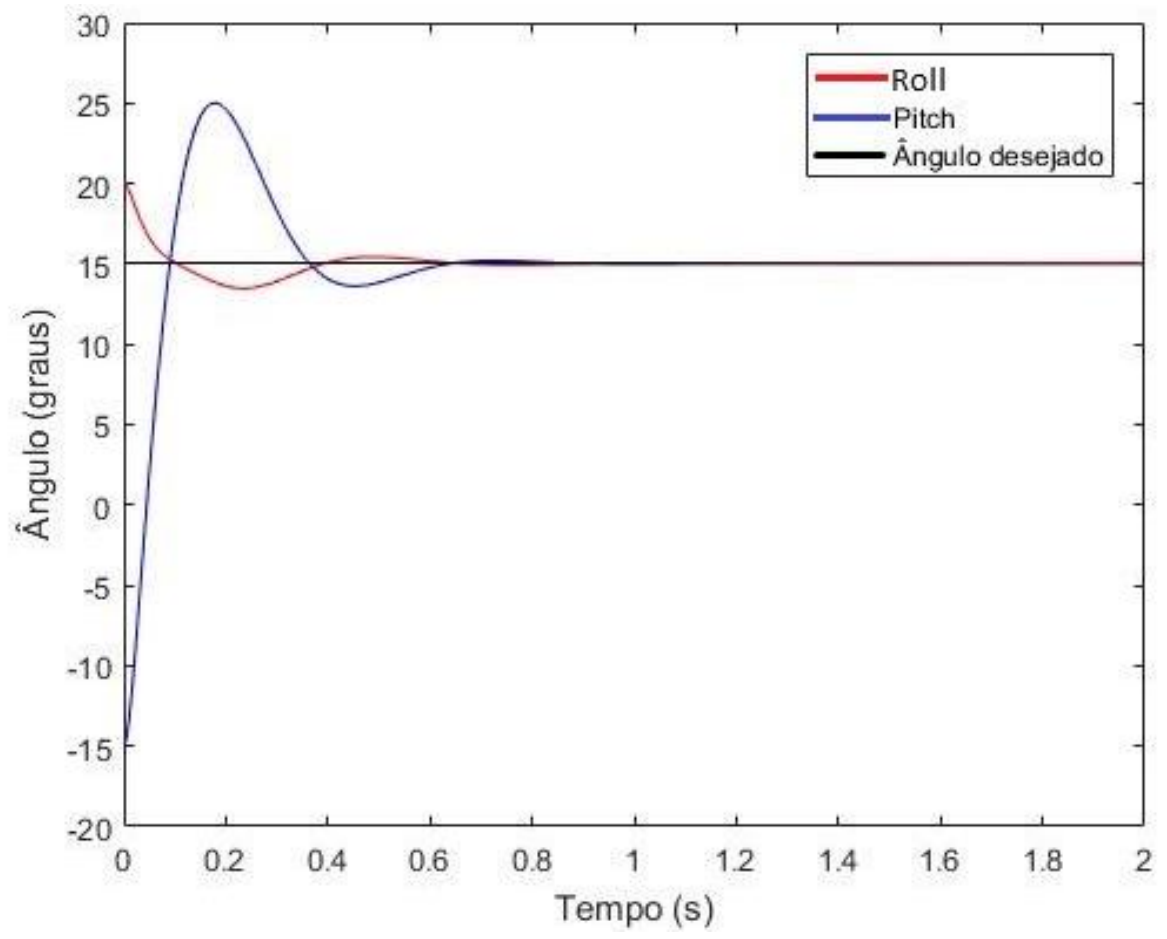

Figura 10 - Roll e pitch em função do tempo, com posição desejada de 15 graus (Fonte: elaborada pelos autores).

Por fim, definiu-se os ângulos iniciais de pitch e roll iguais a 0 graus e posições finais diferentes, sendo 20 graus para pitch e 10 graus para roll. A figura 11 mostra o comportamento do sistema nessa simulação.

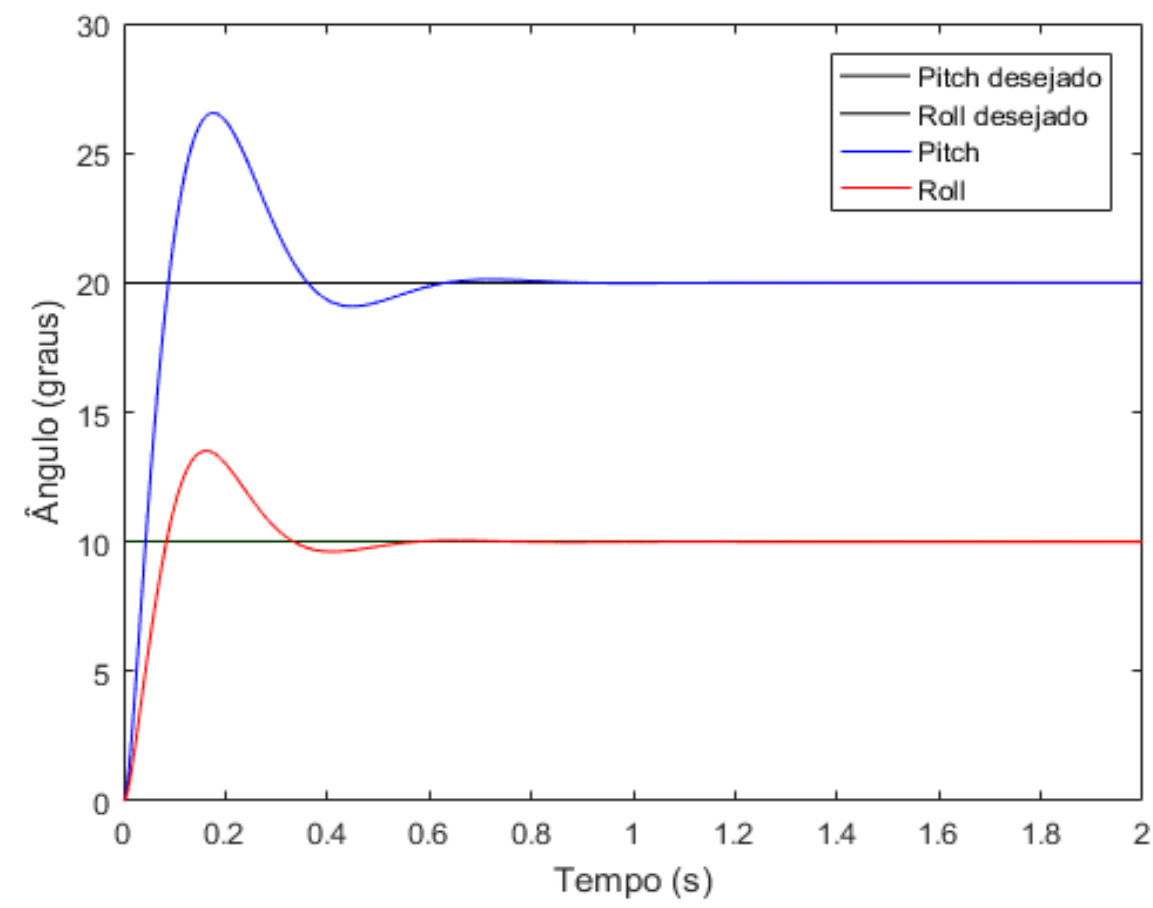

Figura 11 - Roll e pitch em função do tempo, com posições desejadas de 10 e 20 graus, respectivamente (Fonte: elaborada pelos autores). 
Pode-se observar que o sistema respondeu bem às entradas, com uma resposta rápida e eficaz, sendo que conseguiu estabilizar-se em todas as simulações em menos de um segundo.

\subsection{ESTRUTURA}

O CATIA por ser um programa de modelagem é praticável a realização de um modelo da superfície do frame em 3D. Com isso o projetista pode apresentar soluções de visualização de projeto, como por exemplo, buscar perfis com uma aerodinâmica mais eficiente. Deste modo o aluno com base em um modelo de prateleira pode projetar outro frame passando a possuir um conhecimento prático de projeção e compreendendo melhor a estrutura como um todo. A figura abaixo é um modelo do braço do frame em 3D.

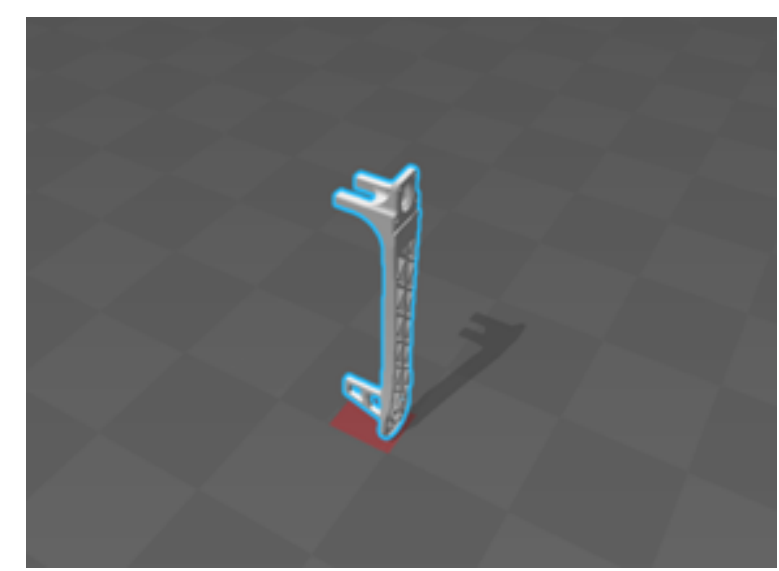

Figura 12: Braço do Frame em 3D (Fonte: [12])

A análise estrutural do dispositivo pode ser feita utilizando o software ANSYS que é capaz de determinar o comportamento da estrutura quando submetida a esforços. Estudandose também as características específicas do comportamento da estrutura quando se aplica materiais diferentes. Podendo- se calcular a deformação na estrutura, reações de apoio, forças internas. Que são dados fundamentais para saber se a estrutura está apta para ser utilizada.

Após esses estudos caso seja conveniente existe a possibilidade ainda de ser reproduzir as peças do frame utilizando uma impressora 3D.

\section{POSSIBILIDADES DE APLICAÇÃO NO ENSINO}

As possibilidades de aplicação do dispositivo são muito amplas e diversificadas. Elas vão de disciplinas do ciclo básico a disciplinas mais avançadas. É possível explorar, por 
exemplo, os conhecimentos básicos aprendidos em Física 1 para calcular o empuxo que o motor consegue gerar e realizar ensaios para verificação e comparação de resultados reais e teóricos. Conceitos de mecânica de fluidos podem ser vistos na prática ao analisar os efeitos que as características das hélices, como tamanho de corda e ângulo de ataque, causam no empuxo.

Conhecimentos de Circuitos Eletrônicos são extremamente necessários, desde a definição dos materiais a serem usados até a montagem. Nesse ponto, é importante ressaltar que o trabalho com o dispositivo mostra ao estudante a necessidade de não apenas saber analisar circuitos eletrônicos, mas também de saber projetar um circuito de forma simples, econômica e funcional. Falhas nos circuitos do dispositivo podem acarretar em grandes perdas (caso o quadrotor fosse realizar voos longos, como em um projeto real), de modo que deve ser incentivada a realização de muitos testes da resposta do circuito em diferentes situações e de resistência a sobrecargas.

Intimamente ligadas aos circuitos eletrônicos estão as habilidades computacionais. Durante o processo de calibragem dos sensores e módulos de comunicação, o conhecimento adquirido em disciplinas de lógica computacional e desenvolvimento de algoritmos é extremamente explorado, sendo ele indispensável neste e em outros processos.

Conhecimentos mais profundos em Física, unidos ao Cálculo e à Álgebra fazem-se necessários para a aplicação das equações de movimento de Newton ao modelo dinâmico do quadrotor. Esse modelo, bem como o domínio de equações diferenciais e habilidades computacionais são fundamentais para o desenvolvimento do sistema de controle do quadrotor. Novamente, assim como no caso dos circuitos eletrônicos, todo esse conhecimento precisa ser unido e aplicado em uma situação real, pois sem ele não é possível a realização desse tipo de projeto. Desse modo, além de estimular o desenvolvimento de atividades práticas, o dispositivo mostra a importância do conhecimento teórico em tais atividades.

A necessidade de interação entre várias áreas do conhecimento em engenharia também proporciona aos estudantes experiências de trabalho em equipe, conferindo-lhes habilidades de gestão, tanto gestão de pessoas, quanto gestão de conhecimento e de projeto. São possíveis ainda muitas outras aplicações do dispositivo no ensino em engenharia. $\mathrm{O}$ objetivo deste artigo não é esgotar as possibilidades de uso da ferramenta, mas demonstrar algumas de suas muitas possibilidades de contribuição no ensino e formação de profissionais engenheiros.

\section{CONCLUSÃO}


Considerando tudo o que foi exposto até aqui, o dispositivo mostra-se eficaz no desenvolvimento de habilidades práticas para futuros engenheiros aeroespaciais e também para graduandos de outras áreas da engenharia, como eletrônica e software. O dispositivo pode ser construído pelos próprios estudantes, ressaltando que todo processo de montagem pode ser alterado, e é interessante que isso aconteça, pois desenvolve habilidades de projeto nos estudantes.

Ainda que já tenha sido citado anteriormente, vale ressaltar que o dispositivo não pode, e nem tem por objetivo, acabar com a dificuldade que os engenheiros recém formados no Brasil encontram ao desenvolver atividades práticas no mercado de trabalho. Entretanto, é uma maneira de tentar amenizar localmente essa dificuldade e, principalmente, incentivar o desenvolvimento de mais alternativas para o treinamento prático de graduandos em engenharia no Brasil. Essas alternativas, acima de tudo, devem ser condizentes com a realidade econômica das universidades brasileiras.

Uma das habilidades que o mercado de trabalho busca em engenheiros é a capacidade de inovar. Eis aqui uma oportunidade para desenvolver esta habilidade. Inovar no ensino, buscar novos caminhos, ver oportunidades em meio às dificuldades. Até a busca por novas alternativas mostra-se como um treinamento para situações exigidas no mercado. Engenheiros que consigam produzir, ainda que as condições sejam desfavoráveis. A dificuldade em manter grandes laboratórios pode ser a oportunidade que o Brasil possui para formar os melhores engenheiros, treinados para buscar alternativas inovadoras, inteligentes e simples.

\section{REFERÊNCIAS}

[1] CNI, Agência de Notícias, Apenas $42 \%$ dos engenheiros brasileiros atuam na área em que se formam. Disponível em:

<http://www.portaldaindustria.com.br/cni/imprensa/2014/04/1,35904/apenas-42-dosengenheiros-brasileiros-atuam-na-area-em-que-se-formam.html>. Acesso em 1 out. 2016

[2] Unmanned Tech, F450 Flaming Wheel Quadcopter Frame. Disponível em: <https://www.unmannedtechshop.co.uk/f450-quadcopter-frame-with-integrated-pdb/>. Acesso em 23 jan. 2017

[3] Art of Circuits, Quadcopter Frame - F450. Disponível em: <http://artofcircuits.com/product/quadcopter-frame-f450>. Acesso em 23 jan. 2017

[4] Solarbotics, Arduino USB Board (UNO R3). Disponível em: $<$ https://solarbotics.com/product/50450/> . Acesso em 24 jan. 2017 
[5] AMB, Combo EMax CF2812 (motor + hélice + ESC 18A).Disponível em:

<http://www.aeromodelosamb.com.br/loja/product_info.php?cPath=45\&products_id=813> . Acesso em 24 jan. 2017

[6] SainSmart, MPU6050 3 Axis Gyroscope Module for Arduino. Disponível em:

<http://www.sainsmart.com/sainsmart-mpu-6050-3-axis-gyroscope-module.html>. Acesso em 24 jan. 2017

[7] Robotech Shop, HC-05 Bluetooth Module. Disponivel em:

<https://robotechshop.com/shop/module/hc-05-bluetooth-module/?v=19d3326f3137>. Acesso em 24 jan. 2017

[8] MATHWORKS. Simulink. Simulink Supported Hardware. Disponível em:

<http://www.mathworks.com/help/simulink/ug/simulink-supported- hardware.html> Acesso em: 20 set. 2015

[9] MATHWORKS. Simulink . Build Arduino projects using high level programming and block diagrams. Disponível em:

$<$ https://www.mathworks.com/discovery/arduino-programming-matlab-simulink.html > Acesso em: 07 out. 2016

[10] National Instruments Corporation, PID Theory explained, 2006, Document version 3.

[11] NoRunway, A Few Flight Related Terms. Disponível em:

<http://norunway.com/wp/flight-related-terms/> Acesso em: 25 jan. 2017

[12] Thingiverse. Disponível em:

<http://www.thingiverse.com/thing:264955> Acesso em: 31 jan. 2017 